ApJ., Revised: 2010-Dec-02

\title{
Outburst of Comet 17P/Holmes Observed With The Solar Mass Ejection Imager
}

\author{
Jing $\operatorname{Li}^{1}$ \\ David Jewitt ${ }^{1,2}$ \\ John M. Clover ${ }^{3}$ \\ and \\ Bernard V. Jackson ${ }^{3}$ \\ (1) Institute for Geophysics and Planetary Physics, University of California at Los Angeles \\ (2) Depts. Earth and Space Sciences and Physics and Astronomy, University of California \\ at Los Angeles \\ (3) Center for Astrophysics and Space Science, University of California at San Diego, La \\ Jolla, CA 92093-0424 \\ jli@igpp.ucla.edu, jewitt@ucla.edu
}

\begin{abstract}
We present time-resolved photometric observations of Jupiter family comet 17P/Holmes during its dramatic outburst of 2007. The observations, from the orbiting Solar Mass Ejection Imager (SMEI), provide the most complete measure of the whole-coma brightness, free from the effects of instrumental saturation and with a time-resolution well-matched to the rapid brightening of the comet. The lightcurve is divided into two distinct parts. A rapid rise between the first SMEI observation on UT 2007 October 24 06h 37m (mid-integration) and UT 2007 October 25, is followed by a slow decline until the last SMEI observation on UT 2008 April 6 22h 16m (mid-integration). We find that the rate of change of the brightness is reasonably well-described by a Gaussian function having a central time of UT 2007 October $24.54 \pm 0.01$ and a full-width-at-half-maximum $0.44 \pm 0.02$ days. The maximum rate of brightening occurs some 1.2 days after the onset of activity. At the peak the scattering cross-section grows at $1070 \pm 40$ $\mathrm{km}^{2} \mathrm{~s}^{-1}$ while the (model-dependent) mass loss rates inferred from the lightcurve reach a maximum at $3 \times 10^{5} \mathrm{~kg} \mathrm{~s}^{-1}$. The integrated mass in the coma lies in the range ( 2 to 90$) \times 10^{10} \mathrm{~kg}$, corresponding to $0.2 \%$ to $10 \%$ of the nucleus mass, while
\end{abstract}


the kinetic energy of the ejecta is (0.6 to 30) MTonnes TNT. The particulate coma mass could be contained within a shell on the nucleus of thickness $\sim 1.5$ to $60 \mathrm{~m}$. This is comparable to the distance travelled by conducted heat in the century since the previous outburst of $17 \mathrm{P} /$ Holmes. This coincidence is consistent with, but does not prove, the idea that the outburst was triggered by the action of conducted heat, possibly through the crystallization of buried amorphous ice.

Subject headings: Comets: general - Comets: individual: 17P/Holmes - Kuiper belt: general

\section{Introduction}

Comet 17P/Holmes is a dynamically unremarkable comet, with a semimajor axis, eccentricity and inclination of $3.620 \mathrm{AU}, 0.433$ and $19.1^{\circ}$, respectively. The Tisserand parameter measured with respect to Jupiter is 2.86 , which classifies $17 \mathrm{P} /$ Holmes as a member of the Jupiter comet family and suggests a likely origin in the Kuiper belt. The perihelion distance is a modest 2.05 AU, small enough to drive the production of a coma through the sublimation of near-surface water ice but large enough that the comet is not normally spectacular as seen from the Earth. As a result, the comet has received relatively little observational attention and the properties of its nucleus are poorly known, except for an estimate of its radius (about $1.7 \mathrm{~km}$, Lamy et al. 2009). However, 17P/Holmes is distinguished by having undergone three dramatic photometric outbursts, the first leading to its discovery in November 1892 (Holmes 1892), followed by an outburst in mid-January 1893 (Barnard 1896), and the most recent being the subject of this paper. The recent outburst was first noticed by J. A. Henriques Santana on UT 2007 October 24.067 (Buzzi et al. 2007) and triggered intensive study by unprecedented numbers of observers around the world. In the course of a day, the comet brightened from about 17th apparent magnitude up to naked-eye visibility, with concurrent expansion of an initially circular coma at the sky-plane velocity $\sim 550 \mathrm{~m} \mathrm{~s}^{-1}$ (Lin et al. 2009 ; Hsieh et al. 2010, corresponding to $\sim 40$ arcseconds day $^{-1}$ at geocentric distance 1.6 AU).

The remarkable photometric characteristics of $17 \mathrm{P} /$ Holmes introduced two practical problems for observers. First, the high initial surface brightness of the coma caused saturation of the data from many instruments, especially those having large apertures and short focal ratios. Second, the expansion of the coma soon over-filled the fields of view of many large telescopes, so that while photometry of the central regions could be obtained, photometry of the whole coma could not.

In this paper, we report observations of $17 \mathrm{P} /$ Holmes fortuitously taken with the Solar 
Mass Ejection Imager (SMEI). This orbiting instrument takes data with a 102 minute cadence well-suited to the study of the temporal evolution of the outbursting comet. Moreover, SMEI images are obtained in such a way that even high surface brightness sources do not lead to saturation of the data, as we will describe. Lastly, the angular resolution of SMEI permits measurements of the integrated light from the whole coma, at least for the first few months. In these several regards, the SMEI data are complementary to other measurements taken with cameras that saturated (Hsieh et al. 2010), or which were unable to image the full coma owing to their limited fields of view (Montalto et al. 2008; Mugrauer et al. 2009; Lin et al. 2009).

\section{Observations}

The Solar Mass Ejection Imager (SMEI) was launched on the Coriolis satellite by the United States Department of Defense in January 2003 (Eyles et al. 2003). The scientific aim of SMEI is to detect and forecast the arrivals of coronal mass ejections (Jackson et al. 2004, Buffington et al. 2008). SMEI has a Sun-synchronous polar orbit above the Earth's terminator, with a period of 102 minutes. Three charge-coupled device (CCD) cameras, each with a field of view $60^{\circ} \times 3^{\circ}$, scan the sky as the satellite orbits the Earth. They are oriented about $20^{\circ}$ above the local horizontal and pointed opposite to the motion of the spacecraft. Their alignments are such that Camera 1 points away from the Sun, Camera 3 points near the Sun and Camera 2 aims in the middle. This allows coverage of nearly the entire sky in one orbit. The camera optics consist of two mirrors behind a complicated baffle structure, with an effective collecting area of $\sim 1.7 \mathrm{~cm}^{2}$.

The image scale of the camera is $0.05^{\circ} \mathrm{pixel}^{-1}$, but is degraded to $0.2^{\circ} \mathrm{pixel}^{-1}$ onboard during normal "science mode" operations. In a normal astronomical camera system, such a large pixel scale would result in rapid saturation of the data from bright stars and even from high surface brightness coronal structures. Two characteristics of the SMEI instruments prevent saturation of detector pixels caused by bright sources in the field of view. First, the exposure time for a single CCD frame is limited to only 4 seconds. Typically 1530 frames are combined from each CCD camera during a single orbit in order to produce one sky map. Second, the images from the camera are intentionally defocussed, such that point sources appear extended and fish-shaped in the plane of the CCD. This reduces the likelihood of saturation by spreading the light from each point source over $\sim 200$ pixels. It also improves the photometric precision (up to $0.1 \%$ ) by allowing a large number of photoelectrons to be

captured in each image without approaching the 350,000 electron full-well capacity of the CCD. The instrument point spread function with a total width of $\sim 1^{\circ}$, provides a $0.1 \%$ 
differential photometric sky brightness response as stellar signals sweep across the camera field of view. The capacity to image bright sources without approaching saturation of the detector is a key advantage of SMEI when used to study outbursting comet 17P/Holmes.

Final images from SMEI are digitally constructed in J2000 equatorial coordinates. The data are re-sampled back to $0.1^{\circ}$ pixel $^{-1}$, to create sky maps for each SMEI camera with dimensions $3600 \times 1200$ pixels in longitude and latitude (Jackson et al. 2004). The processing steps used at UCSD to convert the raw CCD images into photometrically accurate whitelight sky maps include: integration of new data into the SMEI database; removal of an electronic offset (bias) and dark current pattern; identification of cosmic rays, space debris and flipper pixels (see Hick et al. 2005, for further details); and placement of the images onto a high-resolution sidereal grid using spacecraft pointing information. To reduce background subtraction uncertainties, stars brighter than 6th magnitude are automatically removed from SMEI images by fitting the Point-Spread-Function (Hick et al. 2007).

To avoid confusion between the multiple time-systems used to report observations (local time, Universal time, decimal Julian Day numbers, and modified Julian Day numbers have all been used), we employ the Day of Year (DOY) number, defined as being DOY $=1.0$ on UT 2007 January 1 and increasing linearly thereafter (i.e. UT 2008 January 1 is DOY $366)$. In this system, the perihelion of $17 \mathrm{P} /$ Holmes occurred on DOY $=124.6615$, JD = 2454225.1615, UT 2007 May 04.6615.

The first SMEI sky map image showing 17P/Holmes has mid-integration time UT 2007 October 24 06h 37m 02s (DOY 297.275, the sky map was made between 05h 36m 12s and 07h $37 \mathrm{~m} \mathrm{52s)}$ on Camera 1. Two previous images from the same day appear blank, apparently because the shutter of SMEI was closed. The comet is already bright when first recorded and continued to be well-recorded by SMEI Camera 1 to 2008 January 11 08h 36m 26s (mid-time between 07h 35m 36s and 09h 37m 16s) and Camera 2 from UT 2008 January 1 01h $34 \mathrm{~m} 56 \mathrm{~s}$ (mid-time between $00 \mathrm{~h} 43 \mathrm{~m} \mathrm{44s}$ and $02 \mathrm{~h} 26 \mathrm{~m} 08 \mathrm{~s}$ ) to 2008 April $622 \mathrm{~h} 15 \mathrm{~m}$ $58 \mathrm{~s}$ (mid-time between $21 \mathrm{~h} 31 \mathrm{~m} \mathrm{24s}$ and $23 \mathrm{~h} 01 \mathrm{~m} \mathrm{32s}$ ). After April 6, the comet became too faint to be readily measured using SMEI. The observations covered a 165 day period with a total of 1992 sky map images. During this time, the geocentric distance doubled, while the heliocentric distance increased only slightly. The change in the observing geometry is illustrated in Figure 1. Sample SMEI images of 17P/Holmes are shown in Figure 2. 


\section{Analysis}

\subsection{Brightness Calibration}

Data from SMEI are routinely photometrically calibrated using bright stars distributed around the sky. However, because the passband of this filterless instrument is very broad and different from the standard astronomical filters, we elected to calibrate the data against our own measurements of $17 \mathrm{P} /$ Holmes taken nearly simultaneously. For these, we used the University of Hawaii 2.2-m telescope to image 17P/Holmes on UT 2007 October 26.33 $(\mathrm{DOY}=299.33)$ in order to photometrically calibrate the SMEI data. The spectral response of the SMEI imager is very broad, exceeding $10 \%$ in the optical wave band (4500-9500A) and $>40 \%$ over the $6000 \leq \lambda \leq 7500 \AA$ wavelength range. The central wavelength corresponds approximately to the astronomical $R$-band. Accordingly, an $R$-band filter was employed at the 2.2-m telescope and calibrated in the Kron-Cousins photometric system (Landolt 1992). The comet was imaged using a Tektronix $2048 \times 2048$ pixel charge-coupled device camera placed at the f/10 Cassegrain focus, where the plate scale is $0.219^{\prime \prime}$ per pixel and the fieldof-view $450^{\prime \prime} \times 450^{\prime \prime}$. We used aperture photometry with circular projected apertures and experimented to determine the optimum aperture radius for $17 \mathrm{P} /$ Holmes photometry. We found that an aperture radius of 800 pixels $\left(175^{\prime \prime}\right)$ was sufficient to capture $>99 \%$ of the light from the comet on this date. Such a large aperture could not be used to measure the ( 60,000 times fainter) standard star without incurring unacceptable errors from uncertainty in the sky background. Instead, an aperture 20 pixels $\left(4.4^{\prime \prime}\right)$ in radius, with sky determined from the median of data numbers in a surrounding annulus extending to 70 pixels (15.3") radius, was used to measure the Landolt (1992) standard star SA95-98. Again, we checked to be sure that this aperture captured $>99 \%$ of the light from the star.

The particular circumstances of $17 \mathrm{P} /$ Holmes demand special mention here. The high surface brightness of the coma on UT 2007 October 26 forced the use of unusually short integrations. Normally, the Tektronix CCD camera is not used with exposures $<5 \mathrm{~s}$ and, at shorter integration times, the linearity of the shutter (a spring-triggered leaf shutter) is in question. Spatial non-uniformity of the shutter open time with position on the CCD degrades, as does knowledge of the exact duration of the open time. To measure the importance of these effects we compared exposures of 0.1, 0.5, 1.0 and 5.0 seconds to estimate possible photometric errors arising from the forced use of short integrations on $17 \mathrm{P} /$ Holmes. We find that systematic shutter errors are less than $\sim 10 \%$ for the $17 \mathrm{P} /$ Holmes data. This is small enough to be of no significance in the interpretation of the SMEI data. 


\subsection{Photometry of $17 \mathrm{P} /$ Holmes}

The brightness of $17 \mathrm{P} /$ Holmes was measured within projected, circular apertures centered on the photocenter of the object. Use of small apertures is precluded by the large point-spread-function produced by SMEI, while large apertures suffer excessive contamination by background sources. Accordingly, we employed a standard photometry aperture radius of 12 pixels $\left(1.2^{\circ}\right)$ for our measurements, with sky subtraction determined from a contiguous annulus extending to an outer radius of 30 pixels $\left(3.0^{\circ}\right)$ (see Figure 2). We used the median of the pixel values within the sky annulus to define the sky brightness, since the median confers some protection against contamination of the sky brightness by imperfectly removed field stars.

The photometry is shown in Figure 3 as a function of time, with measurements from Cameras 1 and 2 identified. Only Camera 1 measurements were calibrated against (nearly) simultaneous observations from the University of Hawaii telescope. However, the two SMEI cameras provide overlapping coverage in the period $366<D O Y<371$, allowing us to calibrate Camera 2 against Camera 1. Based on this overlap, we have normalized the photometry by subtracting 0.08 mag. from the Camera 2 measurements. Gaps in the lightcurve in Figure 3 appear where field stars have irreversibly compromised the comet data leading to their removal. Remaining excursions in the lightcurve in Figure 3 (e.g. near DOY 340) result from residual contamination of the photometry by the wings of bright, distant stars.

To see the effects of viewing geometry on the lightcurve, we correct the apparent magnitudes, $R$, from Figure 3 , to absolute magnitudes, $R(1,1,0)$, using

$$
R(1,1,0)=R-5 \log _{10}(r \Delta)-2.5 \log _{10} \Phi(\alpha)
$$

in which $r$ and $\Delta$ are the heliocentric and geocentric distances, in AU, and $\Phi(\alpha)$ is the scattering phase function of the comet at phase angle $\alpha$. The phase functions of active comets are difficult to measure because the phase changes are difficult to isolate from simultaneous changes in $R$ and $\Delta$. However, published phase functions are broadly consistent in showing a large forward scattering peak and a more modest back-scattering peak (Millis et al. 1982; Meech \& Jewitt 1987; Schleicher et al. 1998). For this work, we fitted the phase function of Schleicher et al. 1998 (for $0 \leq \alpha \leq 70^{\circ}$ ), to obtain

$$
-2.5 \log _{10} \Phi(\alpha)=0.045 \alpha-0.0004 \alpha^{2}
$$

Near opposition, Equation (2) gives a phase coefficient of order 0.04 mag. degree ${ }^{-1}$, close to the characteristic values measured for the macroscopic surfaces of low albedo aster- 
oids and cometary nuclei ( $\mathrm{Li}$ et al. 2009, and references therein). This suggests that the back-scattering properties of the dust are dominated by particles which are optically large $(2 \pi a / \lambda \geq 1$, or $a \geq 0.1 \mu \mathrm{m}$, given $\lambda \sim 0.6 \mu \mathrm{m})$. This, in turn, is compatible with the optical continuum colors, which are slightly redder than sunlight (Lin et al. 2009), and with inferences from the coma of comet $\mathrm{P} / \mathrm{Halley}$, in which particles with $a<0.1 \mu \mathrm{m}$ were found to contribute negligibly to the integrated scattering cross-section (Lamy et al. 1987). We note that the selection of the particular form of the phase function given by Equation (2) is not critical, since the range of phase angles over which $17 \mathrm{P} /$ Holmes was observed was modest $\left(8.5^{\circ} \leq \alpha \leq 19^{\circ}\right)$ and the effects of phase in Figure 3 are small compared to the effects of the varying geocentric distance.

The resulting absolute magnitudes are shown in Figure 4. Comparison with Figure 3 reveals that the steep decline by about $2.5 \mathrm{mag}$. in apparent brightness observed for DOY $>320$ is largely a geometric artifact. The fading portion of the lightcurve in Figure 4 is comparatively gentle, dimming by only $\sim 0.6$ mag. over the same period. This fading is dominated by the escape of dust particles from the region of the coma sampled by the photometry aperture. Figure 2 shows the change in appearance of the comet in SMEI images resulting from the partial resolution of the expanding coma by the end of 2007 December. Evidence from other observers using smaller apertures confirms this conclusion. For example, Mugrauer et al. (2009) used small aperture photometry and found fading of the apparent magnitude by $\sim 8 \mathrm{mag}$. in the 100 days after the outburst whereas our integrated light photometry shows fading by $\sim 2$ mag. over the same period (Figure 3), almost all of which is due to the changing observing geometry.

\section{Discussion}

\subsection{Scattering Cross-section}

The absolute magnitudes can be used to measure the effective scattering cross-section of $17 \mathrm{P} /$ Holmes, $C_{e}\left(\mathrm{~m}^{2}\right)$, from

$$
p_{R} C_{e}=2.24 \times 10^{22} \pi 10^{0.4\left(R_{\odot}-R(1,1,0)\right)}
$$

in which $p_{R}$ is the geometric albedo measured in the $R$-band and $R_{\odot}=-27.11$ is the apparent red magnitude of the Sun (Russell 1916). We take the geometric albedo of cometary dust to be $p_{R}=0.1$ (Lisse 2002; Hadamcik \& Levasseur-Regourd 2009). Effective cross-sections computed in this way are plotted in Figure 5, where we also show photometry taken in the 
hours preceding the first SMEI observation, by Hsieh et al. (2010). The cross-sections are very large, rising to $C_{e}=5.5 \times 10^{13} \mathrm{~m}^{2}$ by $\mathrm{DOY}=299.25$, and equivalent to a circle of diameter $8.4 \times 10^{6} \mathrm{~m}$, considerably larger than the diameter of the Moon. We note that the SMEI lightcurve in Figure 5 is qualitatively similar to the lightcurve compiled by Sekanina (2008) from visual and other data taken using a wide range of instruments and techniques. However, the latter author derived a peak magnitude $H_{0}=-0.53 \pm 0.12$ from naked eye observations, whereas SMEI data give $R(1,1,0)=-1.8 \pm 0.1$ (see Fig. 5). Part of the difference (perhaps $\sim 0.5$ mag.) can be attributed to the continuum color of the comet (Lin et al. 2009) and the different effective wavelengths of the two measurements. The remainder probably reflects difficulty in using the naked eye to measure the brightness of a diffuse but centrally-condensed source.

The rate of change of the cross-section, $d C_{e} / d t\left(\mathrm{~m}^{2} \mathrm{~s}^{-1}\right)$, is plotted in Figure 6, for a 3-day period containing the start of the outburst. Figure 6 shows that area production peaks at $11.0 \times 10^{8} \mathrm{~m}^{2} \mathrm{~s}^{-1}$ on UT 2007 October $24.54 \pm 0.01$ (DOY $=297.54 \pm 0.01$ ), about 0.5 day before the comet attains peak brightness (and cross-section), as seen in Figure 5 . The peak rate of brightening follows the estimated start of the outburst event (UT 2007 October $23.3 \pm 0.3$, or $\mathrm{DOY}=296.3 \pm 0.3$, Hsieh et al. 2010) by $1.2 \pm 0.3$ days.

\subsection{Optical Depth}

To what extent is the lightcurve in Figures 4 , 5 and 6 influenced by optical depth effects in the coma? The mean scattering optical depth is given by

$$
\bar{\tau}(t)=\frac{C_{e}(t)}{\pi r_{o}(t)^{2}}
$$

where $r_{o}(t)$ is the instantaneous radius of the coma. We write $r_{o}(t)=V\left(t-t_{0}\right)$, where $V=$ $550 \mathrm{~m} \mathrm{~s}^{-1}$ is the expansion speed of the coma and $t_{0}=$ DOY 296.3 is the time of the start of the outburst (Hsieh et al. 2010; Reach et al. 2010). The spatially-averaged optical depth computed from Equations (3) and (4) is plotted in Figure (7), where we see that the peak value in the interval of observations, $\bar{\tau}=3 \times 10^{-3}$, was attained at DOY 297.8, about 1.5 days after the start of the outburst. This does not rule out the possibility that the coma was globally optically thick before it was first observed.

Figure 7 shows that the coma was optically thin, on average, even when at peak brightness, in agreement with the conclusion of Hsieh et al. (2010). Nevertheless, it is still possible that the coma was optically thick when measured along a line to the nucleus, a possibility 
that we address here with a simple model. As a reference point, we assume a spherically symmetric coma in which the number density of dust grains varies with the inverse square of the distance from the nucleus. The line of sight optical depth, in the optically thin limit, then varies as $\tau(p) \propto p^{-1}$, where $p$ is the angle between a given line of sight through the coma and the direction to the nucleus. We write

$$
\tau(p)=\tau_{n}\left[\frac{p_{n}}{p}\right]
$$

where $p_{n}=r_{n} / \Delta$ is the angle subtended by the nucleus radius as observed from Earth and $\tau_{n}$ is the optical depth along a line to the center of the nucleus. We assume $r_{n}=1.7 \mathrm{~km}$ (Lamy et al. 2009) to find, at $\Delta=1.6 \mathrm{AU}, p_{n}=1.5 \times 10^{-3}$ arcsec. The average optical depth across the coma is

$$
\bar{\tau}=\frac{\int_{p_{n}}^{p_{o}} 2 \pi p \tau(p) d p}{\int_{p_{n}}^{p_{o}} 2 \pi p d p}
$$

in which $p_{o}=r_{o}(t) / \Delta$ is the angular radius of the coma at the instant when $\bar{\tau}$ is computed. After substitution and rearrangement, Equations (5) and (6) give

$$
\tau_{n}=\frac{r_{o}(t)}{2 r_{n}} \bar{\tau}
$$

provided $p_{n} \ll p_{o}$.

Equation (7) is a crude approximation in that it assumes spherical symmetry and a $p^{-1}$ coma. Still, to order of magnitude, Equation (7) gives a useful estimate of the likely peak optical depths towards the nucleus. Figure 8 shows that, whereas the average $\bar{\tau}$ is always very small compared to unity, the coma may be optically thick along a line to the nucleus. The nucleus received no direct sunlight as a result of the outburst, proving that the energy driving the expansion was either stored or derived from another source.

The projected angular radius of the optically thick region of the coma is obtained by setting $\tau(p)=1$ in Equation (5), giving $p=\tau_{n} p_{n}$. At the peak $\tau_{n} \sim 65$ (DOY 297.8 from Figure 8), with $p_{n}=0.0015^{\prime \prime}$, we find that the optically thick region subtends an angle $p=$ $0.1^{\prime \prime}$ as seen from Earth. This is comparable to the $\left(\sim 0.06^{\prime \prime}\right)$ angular resolution offered by the best adaptive optics systems on large telescopes, or by the Hubble Space Telescope. It is also so small that the integrated photometric characteristics of $17 \mathrm{P} /$ Holmes are dominated by scattering from the much larger optically thin region of the coma. Our conclusion that 
the coma was optically thick only in a miniscule central region is supported by the optical observations from the UH 2.2-m telescope on UT 2007 October 26 (DOY 299) that were used to photometrically calibrate the SMEI DATA. They show stars undimmed through the coma and a coma surface brightness increasing smoothly with decreasing projected distance from the nucleus. On the other hand, a detection of extinction was reported by Montalto et al. (2008) two days later, on UT 2007 October 28 (DOY 301). They observed the fading of stars, at $3 \sigma$ levels of confidence, separated from the nucleus by $25^{\prime \prime}<p<180^{\prime \prime}$. Our data indicate immeasurably small central optical depths $3 \times 10^{-4}<\tau<2.5 \times 10^{-3}$ at these large projected distances and thus cannot be reconciled with the observations of Montalto et al. (2008).

The above considerations show that optical depth effects play a negligible role in shaping the overall photometric properties of $17 \mathrm{P} /$ Holmes in outburst. Instead, the lightcurve results from both the time dependence of the rate of release of mass (and cross-section) from the nucleus into the coma and the possible evolution of the scattering properties of particles once ejected. Near infrared spectral observations of the inner coma in late October and early November revealed water ice whose sublimation in sunlight would provide a natural mechanism for disaggregating composite grains (Yang et al. 2009). Imaging observations show sub-structure suggestive of the breakup or fragmentation of centimeter- and decimetersized objects ejected from the nucleus of 17P/Holmes (Stevenson et al. 2010). Hsieh et al. (2010) attempted to fit the early portion the lightcurve with a model assuming exponential fragmentation of dust particles and obtained fits with decay timescales of $1000 \mathrm{~s}$ and $2000 \mathrm{~s}$. However, fits to data from their limited $(\sim 4 \mathrm{hr})$ observing window do not match the more extensive SMEI data-set presented here, and any simple model of the lightcurve in terms of dust fragmentation cannot be supported. All we can say based on the lightcurve is that the brightening reflects the combined effects of the time-dependent nucleus mass production function (assumed to be impulsive by Hsieh et al. 2010) and evolutionary changes in the dust scattering properties. The data offer no way to separate these two effects.

\subsection{Mass and Energy}

The conversion between the derived scattering cross-section and the particle mass is model-dependent and very uncertain. The principal unknown is the dust size distribution, but the particle density is also unmeasured and its value must be assumed. The simplest model is to assume that the particles are all spheres of one effective radius, $a_{e}$, and density, $\rho$. Then, the total dust mass is given by 


$$
M=\frac{4}{3} \rho a_{e} C_{e}
$$

Solid spheres scatter electromagnetic radiation most efficiently when $a \sim \lambda($ Bohren \& Huffman 1983). With $a_{e}=\lambda=0.65 \mu \mathrm{m}$ and $\rho=400 \mathrm{~kg} \mathrm{~m}^{-3}$, the peak $C_{e}=5.5 \times 10^{13} \mathrm{~m}^{2}$ (Figure (5)) gives mass $M=1.9 \times 10^{10} \mathrm{~kg}$. The mass of the nucleus, taken to be a sphere of radius $1.7 \mathrm{~km}$ and having the same density, is $M_{n}=8 \times 10^{12} \mathrm{~kg}$, so that $M / M_{n} \sim 0.2 \%$.

However, this simplest case is likely to underestimate the dust mass, because the real particles will occupy a size distribution in which large particles might contain significant mass while presenting negligible cross-section. Optical data alone provide little or no evidence concerning such particles but we can estimate an upper limit to the dust mass as follows. The spectral energy distribution from optical $(0.5 \mu \mathrm{m})$ to mid-infrared $(20 \mu \mathrm{m})$ wavelengths has been modelled in $17 \mathrm{P} /$ Holmes in terms of power-law distributions of dust particles size in which the number of particles having radius between $a$ and $a+d a$ is proportional to $a^{-q} d a$ (Ishiguro et al. 2010). The models indicate $q>3$ over the radius range $0.3 \mu \mathrm{m}$ to $100 \mu \mathrm{m}$. Measurements in other disintegrating comets show that, while the size distribution is not precisely described by a power law of any index, the data are broadly compatible with power law models $3<q<4$ (Fuse et al. 2007; Jewitt et al. 2010; Vaubaillon \& Reach 2010). We consider a middle value, $q=3.5$, with minimum and maximum particle radii, $a_{1}$ and $a_{2}$, respectively. The effective radius is then $a_{e}=\left(a_{1} a_{2}\right)^{1 / 2}$. With $a_{1}=0.1 \mu \mathrm{m}$ (particles much smaller than this have negligible interaction with optical photons and so present no cross-section for scattering) and $a_{2}=10^{-2} \mathrm{~m}$ (Grün et al. 2001), we obtain $a_{e}=\left(a_{1} a_{2}\right)^{1 / 2}=$ $30 \mu \mathrm{m}$. The mass computed from Equation (8) then rises to $M=9 \times 10^{11} \mathrm{~kg}$, or $M / M_{N} \sim$ $10 \%$. The range of inferred dust masses, $2 \times 10^{10}<M<90 \times 10^{10} \mathrm{~kg}$, may be compared with the best estimate from mid-infrared thermal observations, namely $M=1.0 \times 10^{10} \mathrm{~kg}$ (Reach et al. 2010).

Masses near the upper limit, $2 \times 10^{11} \mathrm{~kg}$, have been claimed based on millimeter wavelength radio-continuum measurements (Altenhoff et al. 2009). We have reinterpreted these measurements according to the formalism described in Jewitt \& Luu (1992). The principal uncertainty is the opacity. With $\lambda=1 \mathrm{~mm}$ opacities in the range 1 to $10 \mathrm{~m}^{2} \mathrm{~kg}^{-1}$, we estimate dust masses from the radio-continuum in the range $10^{9}$ to $10^{10} \mathrm{~kg}$ on UT 2007 October 27.1 within a $5.7^{\prime \prime} \times 7.3^{\prime \prime}$ beam. These masses are 1 to 2 orders of magnitude smaller than derived by Altenhoff et al. (2009), but consistent with a re-analysis of the same radio-continuum data by Reach et al. (2010) and with the range of masses allowed by the SMEI photometry alone. Unfortunately, even the earliest reported radio-continuum measurements sample only a tiny central region in the expanding coma. For example, the first radio-continuum measurement on UT Oct 27.105 used an elliptical $5.7^{\prime \prime} \times 7.3^{\prime \prime}$ beam at a time $(\sim 4$ days after 
the outburst) when the angular diameter of the dust coma was already $300^{\prime \prime}$. Therefore, the radio-continuum data on $17 \mathrm{P} /$ Holmes offer only a lower limit to the total dust mass.

For the rest of the discussion, we use $2 \times 10^{10}<M<90 \times 10^{10} \mathrm{~kg}$ as the best estimate of the dust mass. From Equation (8) the rate of dust production by mass is $d M / d t=$ $4 / 3 \rho a_{e}\left(d C_{e} / d t\right)$, giving $d M / d t \sim(3$ to 140$) \times 10^{5} \mathrm{~kg} \mathrm{~s}^{-1}$ at the maximum on UT 2007 October $24.54 \pm 0.01$ (DOY 297.54 \pm 0.01 ). No contemporaneous measurements of the gas production rate are available. The earliest reported gas production rate is by Combi et al. (2007), who measured $Q\left(H_{2} O\right)=1.4 \times 10^{30} \mathrm{~s}^{-1}$ on UT 2007 October 27 (DOY 300), corresponding to $0.4 \times 10^{5} \mathrm{~kg} \mathrm{~s}^{-1}$. Some 4 days after the start of the outburst and 3 days past its peak, the dust production at this time was already negligible (Figure 6). Schleicher (2009) extrapolated narrowband photometry data to infer peak water production rates $Q\left(H_{2} O\right) \sim 7 \times 10^{29} \mathrm{~s}^{-1}$, or $0.2 \times 10^{5} \mathrm{~kg} \mathrm{~s}^{-1}$.

\subsection{Ejecta and Outburst Trigger}

The ejected dust mass is equivalent to a cube having a side length $(M / \rho)^{1 / 3}=370 \mathrm{~m}$ to $1300 \mathrm{~m}$, where $\rho=400 \mathrm{~kg} \mathrm{~m}^{-3}$ is the assumed bulk density of the nucleus. However, this is an unlikely description of the outburst geometry, for three reasons. Firstly, the skyplane morphology of $17 \mathrm{P} /$ Holmes was initially circularly symmetric, with global deviations from circularity only appearing on timescales of a week after radiation pressure had begun to deflect coma dust (Stevenson et al. 2010). Eruption of material from a localized surface source would more naturally produce a jet or a cone, not a spherical debris cloud or one that appeared symmetric in projection onto the sky. (It is sometimes argued that projection effects would hide deviations from circular symmetry because of the small phase angles of observation but, in fact, with an average phase angle $\sim 0.2$ radian, (see Figure 1) any strong asymmetries would easily have been detected if present). Secondly, the collimated ejection of mass from a spatially localized source would impart significant recoil to the motion of the nucleus. Very roughly, the velocity impulse on the nucleus is given by $\Delta V=\left(M / M_{N}\right) V$, where $V=550 \mathrm{~m} \mathrm{~s}^{-1}$ is the ejecta velocity. Substituting $0.2 \%<M / M_{N}<10 \%$ gives $\Delta V=$ 1.5 to $70 \mathrm{~m} \mathrm{~s}^{-1}$. In one month, an impulse of this magnitude would lead to a displacement of the nucleus from its pre-outburst predicted position by $3900 \mathrm{~km}$ to $180,000 \mathrm{~km}$, and this could scarcely have escaped detection. (At our request, Dr. Brian Marsden examined the reported positions of $17 \mathrm{P} /$ Holmes before and after the outburst to search for evidence of a change in the fitted non-gravitational parameters, but found none). Lastly, on physical grounds it is difficult to imagine a process that would drive mass-loss many hundreds of meters deep into the nucleus against the expected radial gradient of temperature from the 
hot surface to the cold interior.

At the other extreme, the ejected mass could be contained in a surface layer on the nucleus having thickness

$$
\ell=\frac{M}{4 \pi r_{n}^{2} f \rho},
$$

where $f$ is the fraction of the surface area of the nucleus that is ejected. Substituting $f=$ 1 gives $1.4 \mathrm{~m}<\ell<60 \mathrm{~m}$. Shell-like models have been championed for comets including 17P/Holmes for many years (Sekanina 1982, 2008, 2009). In these models, the rapid increase in brightness and scattering cross-section would be caused by disaggregation of the shell, presumably driven by sublimation of ices acting as glue in aggregated structures when freshly exposed to solar radiation and by collisions between disaggregated pieces moving at different speeds under gas drag near the nucleus. Sekanina's model is not contradicted by any aspect of the SMEI photometry. In this scenario, the $1.2 \pm 0.3$ day lag between the start of the outburst and the peak rate of area production (Figure (6)) provides a measure of the timescale of the disaggregation.

We compare $\ell$ with the distance over which heat can be transported in the nucleus by conduction. From solution of the heat diffusion equation, this distance is $\delta r=(\kappa P / \pi)^{1 / 2}$, where $\kappa$ is the thermal diffusivity of the surface materials and $P$ is the period of time over which conduction acts. The thermal diffusivity of porous dielectrics is roughly $\kappa \sim 10^{-7}$ $\mathrm{m}^{2} \mathrm{~s}^{-1}$. For example, setting $P=6.88 \mathrm{yr}$, the orbital period of $17 \mathrm{P} /$ Holmes, we find, $\delta r$ $=2.5 \mathrm{~m}$. Periodic forcing of the insolation as the nucleus moves around its eccentric orbit drives a wave of conducted heat into the nucleus that damps over a length scale $\delta r \sim 2.5$ m. In the $\sim 100$ yrs elapsed since the outbursts of $1892 / 93$, conducted heat would reach $\delta r \sim 25 \mathrm{~m}$ beneath the initial surface. Regions with depth $\gg \delta r$ will be largely immune to surface heating effects driven by recent surface events and thus are candidate locations for the survival of amorphous and trapped supervolatile ices. An important conclusion is that, within (considerable) uncertainties, $\ell \sim \delta r$. This approximate equality suggests that the outburst of $17 \mathrm{P} /$ Holmes in 2007 could have been triggered by heat conducted from the surface first exposed to space and direct sunlight by the outbursts of 1892/93.

A plausible trigger is the crystallization of amorphous water ice, which is exothermic and which is expected to result in the release of trapped supervolatile gases capable of driving the outburst (Prialnik et al.|2004: Bar-Nun et al.|2007; Reach et al. 2010). Amorphous ice has not been directly detected, but provides a self-consistent explanation for the activity observed in comets (Meech et al. 2009) and Centaurs (Jewitt 2009) located beyond the orbit of Jupiter (where temperatures are too low for crystalline water ice to sublimate). Crystallization 
models of comets necessarily assume values for many unknown or poorly-constrained physical parameters (e.g. the thermal diffusivity, the ice/rock ratio, the nucleus spin properties, the mass of trapped gas, even the orbital evolution in the recent past is important in determining the subsurface temperature structure). As a consequence, crystallization models are very flexible but also very difficult to reject based on observations. One feature that is largely independent of the many unknowns is the step-wise progression of the crystallization front into the nucleus. Thermal runaways triggered by crystallization near the surface propagate downward into colder ice. Eventually, the heat released by crystallization is insufficient to drive additional ice to crystallize, and the runaway stops. The vertical distance is related to the thermal skin depth impressed in the nucleus by sunlight added at the surface and typically measured in meters. Crystallization is therefore at least qualitatively consistent with a scenario in which a disintegrating dusty surface shell is launched from the $17 \mathrm{P} /$ Holmes nucleus.

Kossacki \& Szutowicz (2010) computed thermal models of 17P/Holmes and reached the opposite conclusion, namely that runaway crystallization is unlikely to have been responsible for the outburst. However, their conclusion relied, in part, on the very high ejected mass estimates of $10^{12}$ to $10^{14} \mathrm{~kg}$ by Montalto et al. (2008). As noted earlier, the latter mass estimates are based on a reported detection of extinction in the coma which sits uncomfortably with the large-aperture SMEI data presented here. In fact, the upper end of the Montalto et al. (2008) mass estimate considerably exceeds our best guess as to the mass of the entire nucleus of $17 \mathrm{P} /$ Holmes, and therefore cannot be correct. For this reason, and because our own mass estimates (see also Sekanina 2008; Ishiguro et al. 2010, Reach et al. 2010) are considerably smaller, we consider that to reject crystallization as the energy source for the 17P/Holmes outburst would be premature.

If the particles all travel with characteristic speed $V=550 \mathrm{~m} \mathrm{~s}^{-1}$, their total kinetic energy is $E=(3$ to 140$) \times 10^{15} \mathrm{~J}$, equivalent to about 0.7 to 33 Megaton of TNT ( $1 \mathrm{MT}=$ $\left.4.2 \times 10^{9} \mathrm{~J}\right)$. This, in turn, is equal to the total solar energy falling on the $1.7 \mathrm{~km}$ radius nucleus in 7 to 350 days. While sunlight might be needed to trigger the outburst of $17 \mathrm{P} /$ Holmes, it clearly cannot supply enough energy to drive it. The crystallization of amorphous water ice releases $\Delta E \sim 9 \times 10^{4} \mathrm{~J} \mathrm{~kg}^{-1}$. Curiously, this is close to the energy per unit mass of the $17 \mathrm{P} /$ Holmes coma, $E / M \sim 10^{5} \mathrm{~J} \mathrm{~kg}^{-1}$. Therefore, crystallization of a subsurface layer of amorphous ice with the associated release of trapped supervolatile gases could supply the mass, energy and momentum of the ejecta responsible for the remarkable outburst of comet $17 \mathrm{P} /$ Holmes. However, why $17 \mathrm{P} /$ Holmes should be uniquely afflicted by three such extraordinary outbursts, whereas most other comets show none, remains a complete mystery. 


\section{Summary}

We have used photometric time-series data from the orbiting Solar Mass Ejection Imager to study the evolution of outbursting comet 17P/Holmes. The SMEI's large pixel size and the broad field of view allow the spatially and photometrically full coverage of the comet during its rapid expansion due to outburst.

1. The comet was first detected by SMEI on UT 2007 October 24.275 (DOY 297.275) at apparent red magnitude 4.25, quickly brightened to peak brightness (apparent red magnitude 1.8) over the following day and thereafter faded over the next 5 months.

2. The coma remained globally optically thin (average optical depth $<3 \times 10^{-3}$ ) at all times but is inferred to have been locally optically thick (on a line of sight to the nucleus) through the period of observations.

3. The mass of the dust coma was ( 2 to 90$) \times 10^{10} \mathrm{~kg}$, corresponding to $0.2 \%$ to $10 \%$ of the nucleus mass. The ejected mass is equivalent to that contained within a surface shell on the $1.7 \mathrm{~km}$ radius nucleus having a thickness $1.4 \mathrm{~m}$ to $60 \mathrm{~m}$. Comparison with the $\sim 25 \mathrm{~m}$ thermal skin depth for heat conducted inwards since the previous outbursts in $1892 / 93$, is consistent with conducted heat being the trigger responsible for the outbursts.

4. The rate of change of the scattering cross-section can be approximately matched by a Gaussian function having mid-time UT 2007 October 24.54 \pm 0.01 (DOY 297.54 \pm 0.01 ) and full-width at half-maximum $0.44 \pm 0.02$ days. Thus, there is a $1.2 \pm 0.3$ day lag between the start of the outburst (as inferred from observations by Hsieh et al. 2010) and the time of peak activity that may measure the timescale for the disintegration of fragments in the coma. Dust cross-section was added to the coma at a peak rate of $1070 \pm 40 \mathrm{~km}^{2} \mathrm{~s}^{-1}$.

5. The kinetic energy of the outburst was in the range $(3$ to 140$) \times 10^{15} \mathrm{~J}$, far too large for sunlight to play any more than a triggering role in the expansion of the ejecta. The energy per unit mass of the ejecta $\left(10^{5} \mathrm{~J} \mathrm{~kg}^{-1}\right)$ is of the same order as the energy per unit mass released upon the crystallization of amorphous water ice.

The comet Holmes observation by SMEI was first brought to our attention by the web site: http://www.smei.nso.edu/gallery.html sponsored by Air Force Research Laboratory, Space Weather Center of Excellence, National Solar Observatory. We thank SMEI team 
member Dr. Pierre Hick for his generous contribution to the data processing description and Dr. Andrew Buffington for helpful discussions. We thank the referee and Michal Drahus for comments which helped to improve the presentation. SMEI was designed and constructed by a team of scientists and engineers from the US Air Force Research Laboratory, the University of California at San Diego, Boston College, Boston University, and the University of Birmingham, UK. This work was supported, in part, by grants to DJ from NASA's Planetary Astronomy and Outer Planets Research programs. 


\section{REFERENCES}

Altenhoff, W. J., Kreysa, E., Menten, K. M., Sievers, A., Thum, C., \& Weiss, A. 2009, A\&A, 495,975

Bar-Nun, A., Notesco, G., \& Owen, T. 2007, Icarus, 190, 655

Barnard, E. E. 1896, ApJ, 3, 41

Bohren, C. F., \& Huffman, D. R. 1983, Absorption and scattering of light by small particles (New York: Wiley)

Buffington, A., Bisi, M. M., Clover, J. M., Hick, P. P., Jackson, B. V., \& Kuchar, T. A. 2008, ApJ, 677, 798

Buzzi, L., Muler, G., Kidger, M., Henriquez Santana, J. A., Naves, R., Campas, M., Kugel, F., \& Rinner, C. 2007, IAU Circ., 8886, 1

Combi, M. R., Maekinen, J. T. T., Bertaux, J.-L., Quemerais, E., \& Ferron, S. 2007, IAU Circ., 8905, 1

Eyles, C. J., et al. 2003, Sol. Phys., 217, 319

Fuse, T., Yamamoto, N., Kinoshita, D., Furusawa, H., \& Watanabe, J.-I. 2007, PASJ, 59, 381

Grün, E., et al. 2001, A\&A, 377, 1098

Hadamcik, E., \& Levasseur-Regourd, A. C. 2009, Planet. Space Sci., 57, 1118

Hick, P., Buffington, A., \& Jackson, B. V. 2005, Proc. SPIE, 5901, 340

Hick, P., Buffington, A., \& Jackson, B. V. 2007, Proc. SPIE, 6689

Holmes, E. 1892, The Observatory, 15, 441

Hsieh, H. H., Fitzsimmons, A., Joshi, Y., Christian, D., \& Pollacco, D. L. 2010, MNRAS, 407,1784

Ishiguro, M., et al. 2010, ApJ, 714, 1324

Jackson, B. V., et al. 2004, Sol. Phys., 225, 177

Jewitt, D. 2009, AJ, 137, 4296 
Jewitt, D., \& Luu, J. 1992, Icarus, 100, 187

Jewitt, D., Weaver, H., Agarwal, J., Mutchler, M. and Drahus, M. 2010, Nature, 467, 817-819

Kossacki, K. J., \& Szutowicz, S. 2010, Icarus, 207, 320

Lamy, P. L., Gruen, E., \& Perrin, J. M. 1987, A\&A, 187, 767

Lamy, P. L., Toth, I., Weaver, H. A., A’Hearn, M. F., \& Jorda, L. 2009, A\&A, 508, 1045

Landolt, A. U. 1992, AJ, 104, 340

Li, J.-Y., A’Hearn, M. F., Farnham, T. L., \& McFadden, L. A. 2009, Icarus, 204, 209

Lin, Z.-Y., Lin, C.-S., Ip, W.-H., \& Lara, L. M. 2009, AJ, 138, 625

Lisse, C. 2002, Earth Moon and Planets, 90, 497

Meech, K. J., \& Jewitt, D. C. 1987, A\&A, 187, 585

Meech, K. J., et al. 2009, Icarus, 201, 719

Millis, R. L., Ahearn, M. F., \& Thompson, D. T. 1982, AJ, 87, 1310

Montalto, M., Riffeser, A., Hopp, U., Wilke, S., \& Carraro, G. 2008, A\&A, 479, L45

Mugrauer, M., Hohle, M. M., Ginski, C., Vanko, M., \& Freistetter, F. 2009, Astronomische Nachrichten, 330, 425

Prialnik, D., Benkhoff, J., \& Podolak, M. 2004, Comets II, University of Arizona Press, Tucson, p359

Reach, W. T., Vaubaillon, J., Lisse, C. M., Holloway, M., \& Rho, J. 2010, Icarus, 208, 276

Russell, H. N. 1916, ApJ, 43, 173

Schleicher, D. G., Millis, R. L., \& Birch, P. V. 1998, Icarus, 132, 397

Schleicher, D. G. 2009, AJ, 138, 1062

Sekanina, Z. 1982, In: Comets,ed. L. L. Wilkening, Tucson, AZ, University of Arizona Press, p. $251-287$

Sekanina, Z. 2008, International Comet Quarterly, 30, 3

Sekanina, Z. 2009, International Comet Quarterly, 31, 99 
Stevenson, R., Kleyna, J., \& Jewitt, D. 2010, AJ, 139, 2230

Vaubaillon, J. J., \& Reach, W. T. 2010, AJ, 139, 1491

Yang, B., Jewitt, D., \& Bus, S. J. 2009, AJ, 137, 4538 


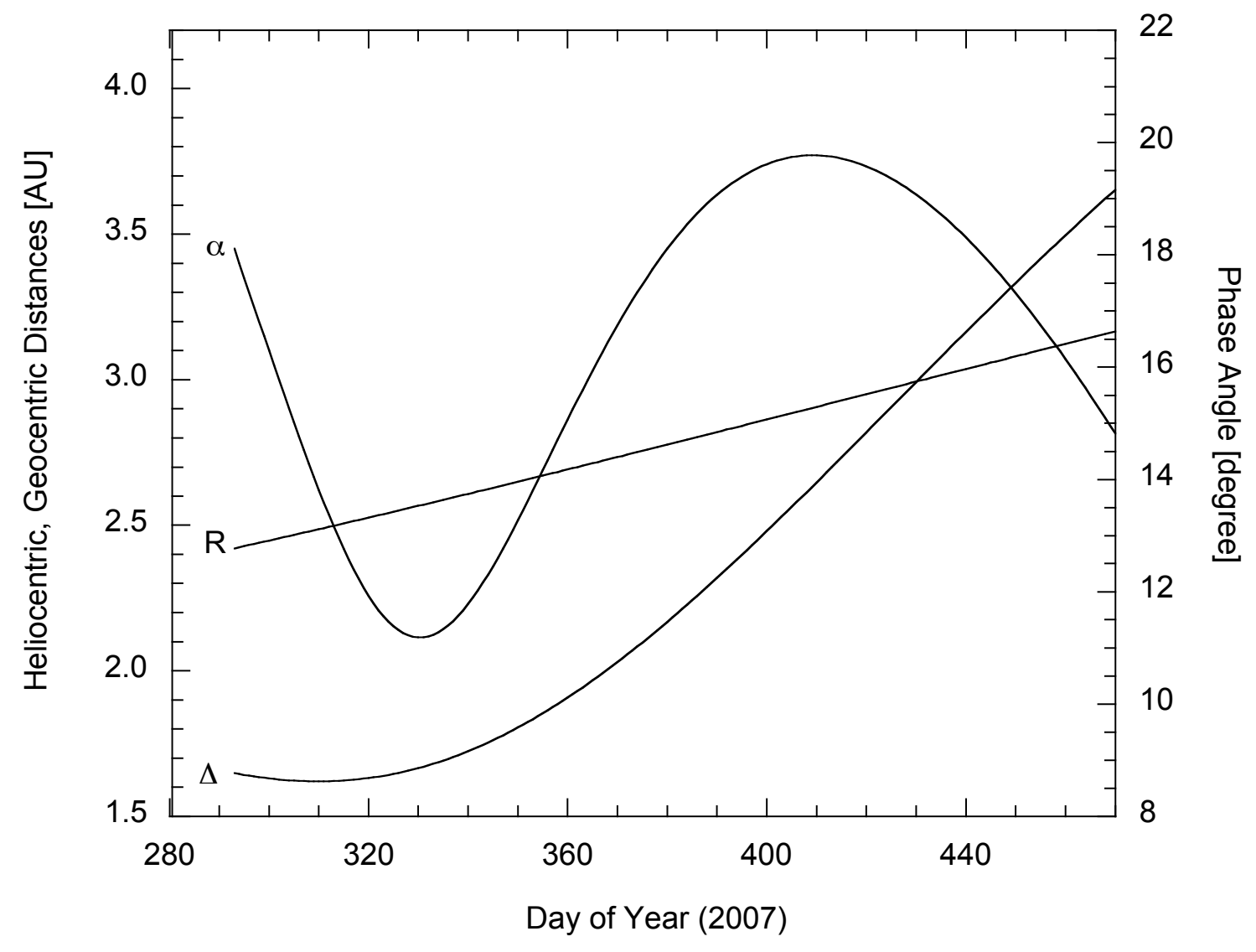

Fig. 1.- (left axis) Heliocentric and geocentric distances of $17 \mathrm{P} /$ Holmes, $R$ and $\Delta$ respectively, and (right axis) phase angle, $\alpha$, as functions of time, expressed as day-of-year in 2007. 


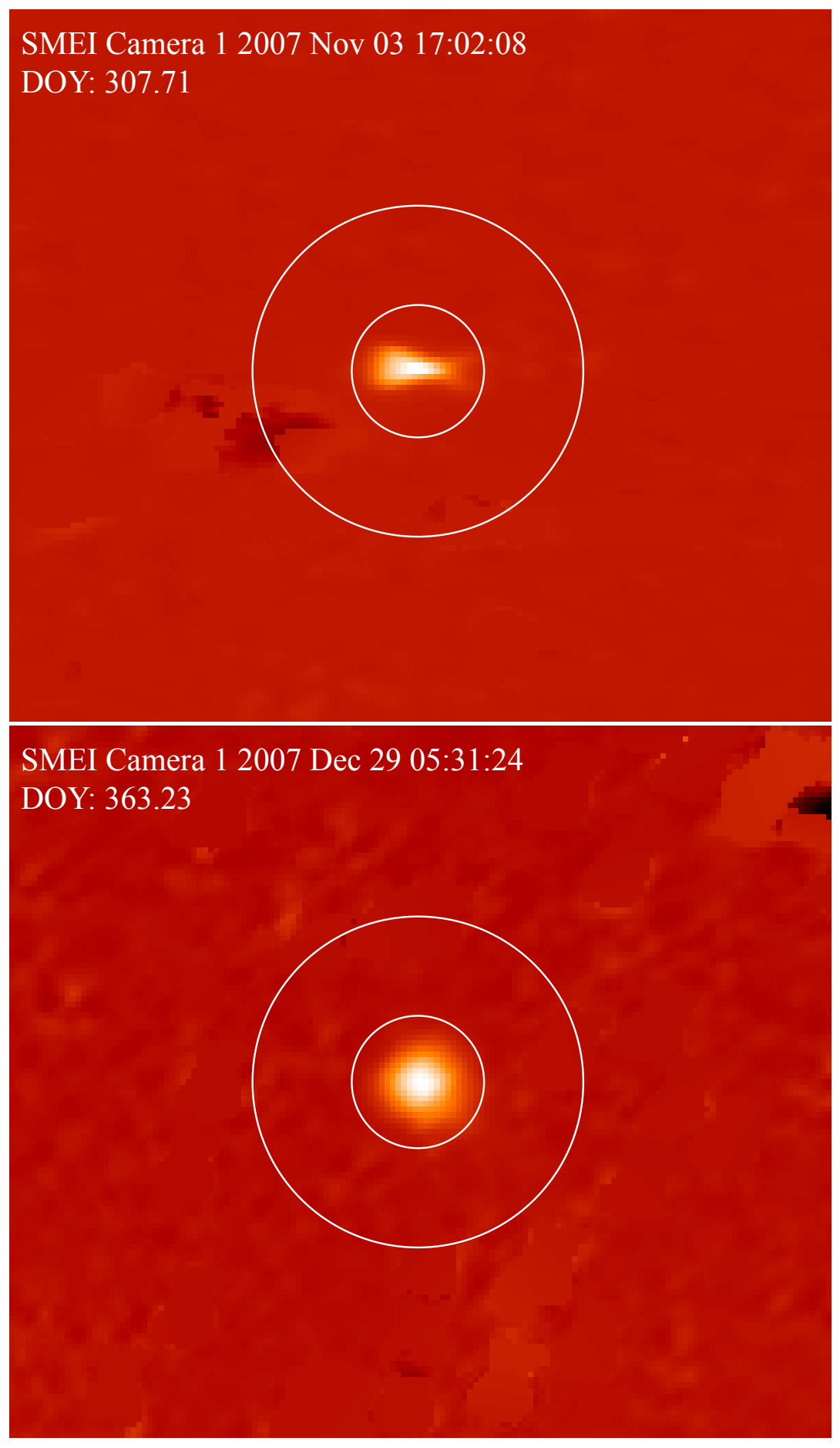

Fig. 2.- Sample images of 17P/Holmes from SMEI Camera 1 taken 2007 November 3 (top) and December 29 (bottom). The region shown in each panel is $14.9^{\circ} \times 12.9^{\circ}(149 \times 129$ pixels) across with North to the top and East to the left. Background stars brighter than 6th magnitude have been removed. The circles around comet $17 \mathrm{P} /$ Holmes have radii $1.2^{\circ}$, and $3.0^{\circ}$, respectively. On November 3, Holmes was unresolved, showing the intrinsic, fish-like SMEI image shape (top). By the end of December 2007, 17P/Holmes was partially resolved by SMEI so that the image appears more as a fuzzy ball (bottom). 


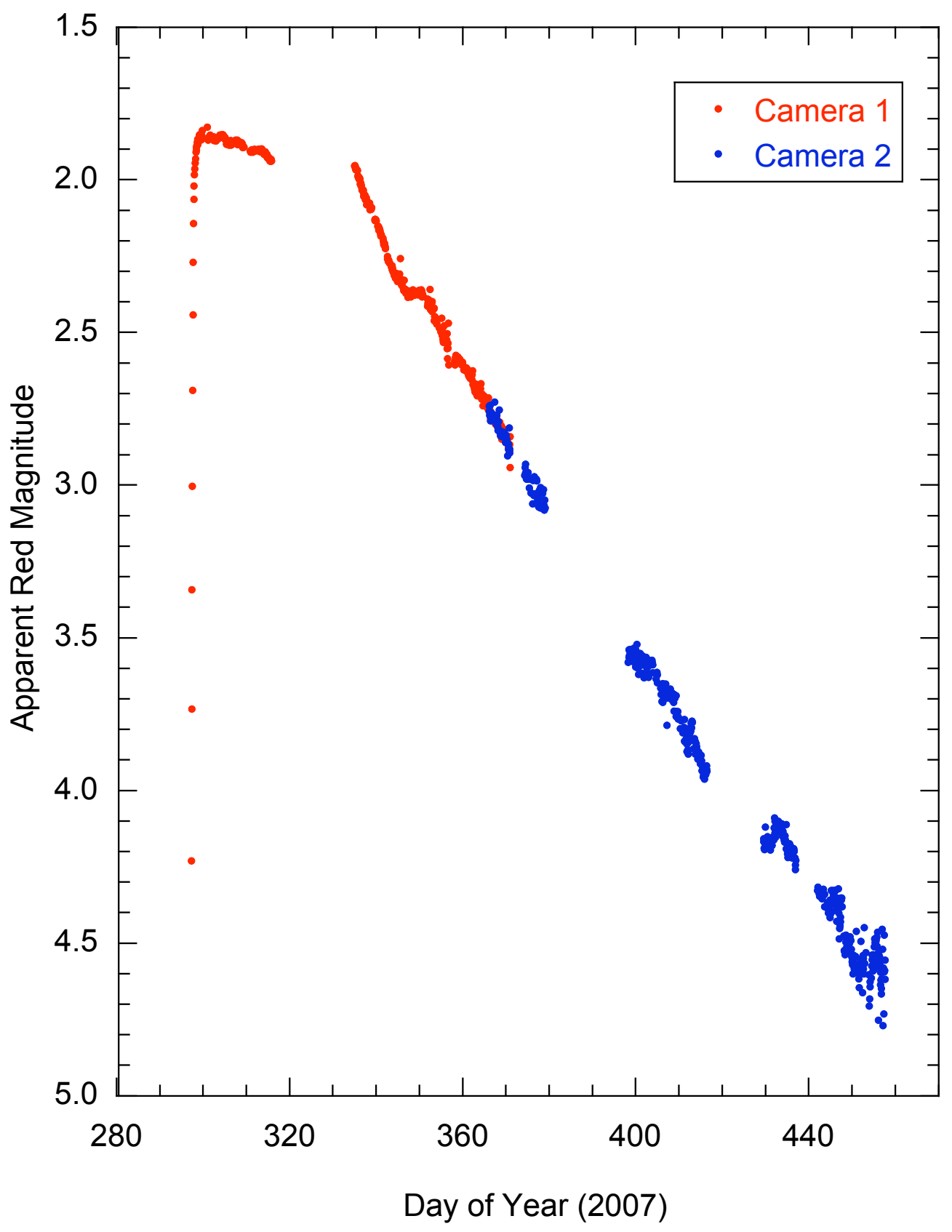

Fig. 3.- Lightcurve of 17P/Holmes deduced from SMEI data. Gaps in the data show where bright field stars contributed excessive contamination. 


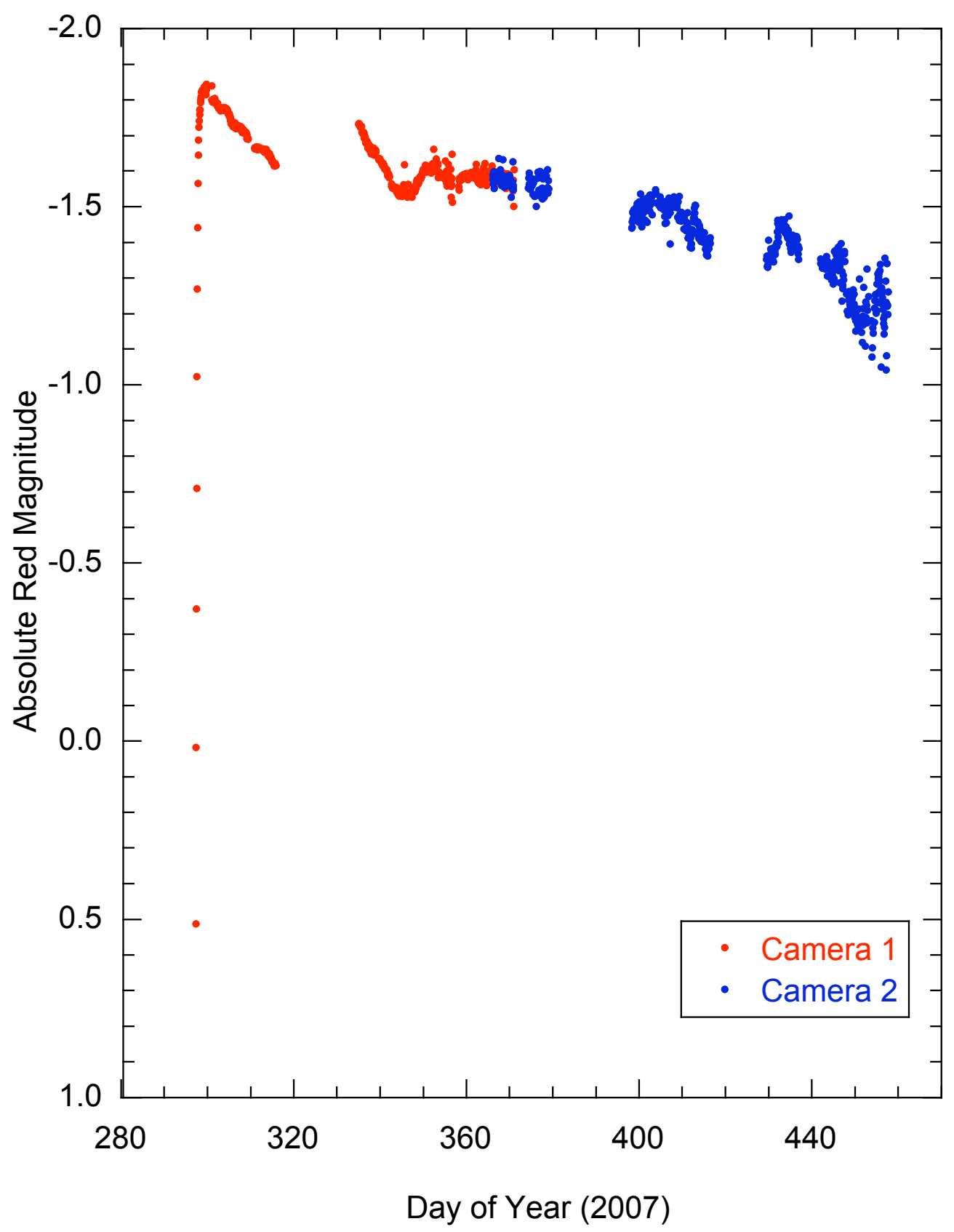

Fig. 4.- The lightcurve of $17 \mathrm{P} /$ Holmes corrected for the effects of changing observing geometry and normalized to unit heliocentric and geocentric distances, and to zero phase angle. Gaps in the data show where bright field stars contributed excessive contamination. 


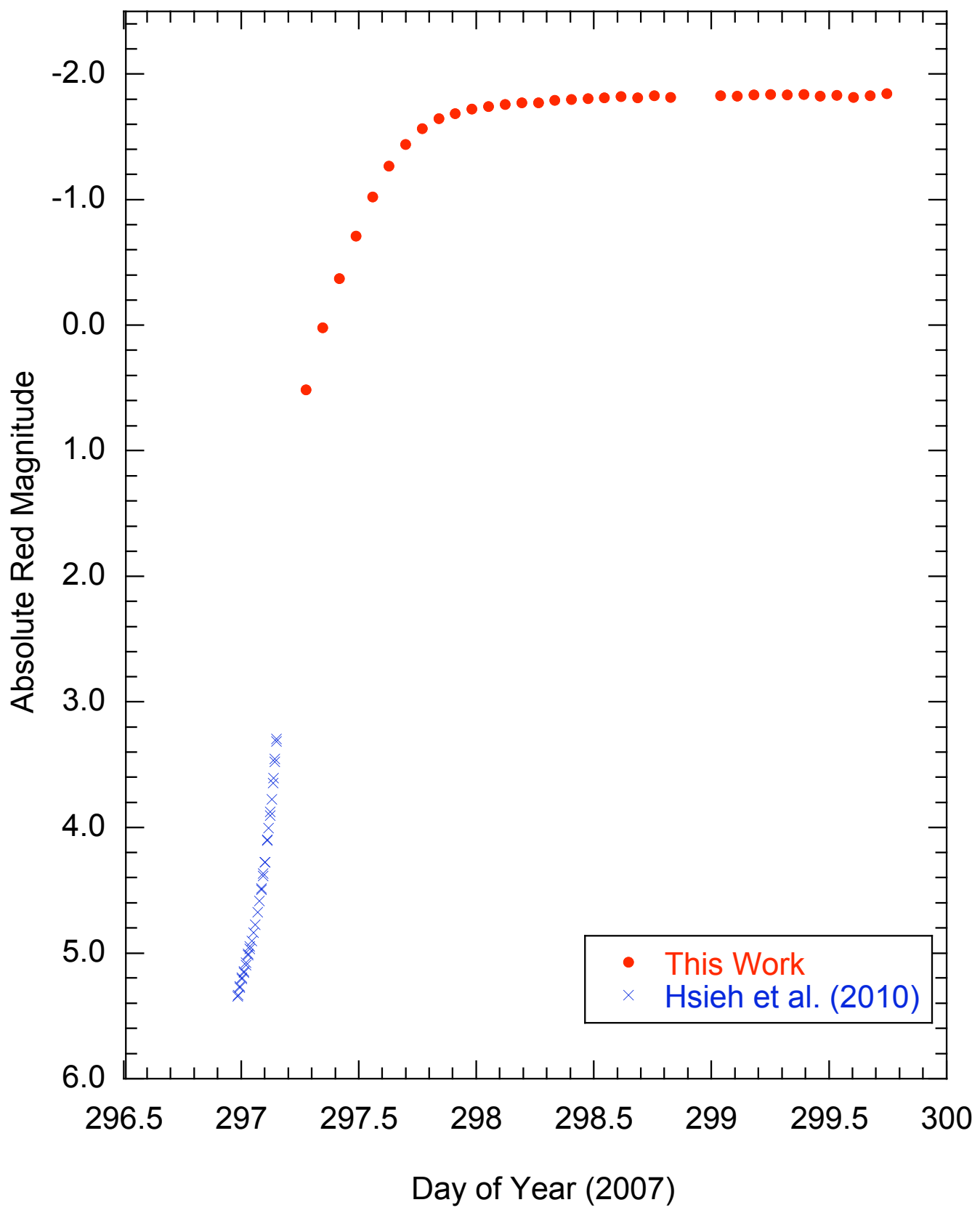

Fig. 5.- Absolute lightcurve from SMEI data compared with data from SuperWASP (Hsieh et al. 2010). 


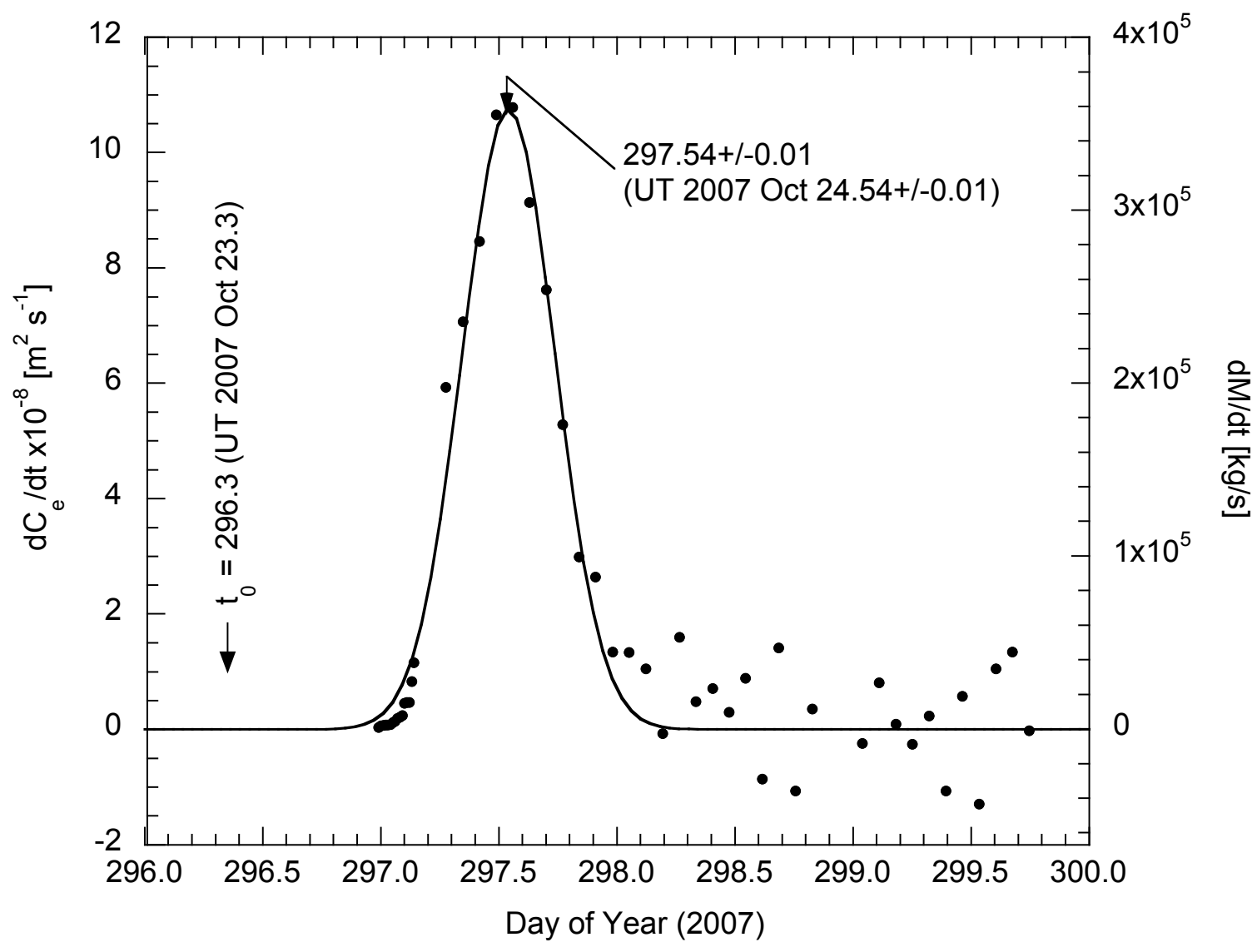

Fig. 6. - (left) Rate of change of scattering cross-section and (right) rate of change of dust mass, as functions of time. The solid line shows a Gaussian function fitted to the data. The approximate time of the start of the outburst, $t_{0}=296.3$, is indicated. The mass production rate refers to an effective particle size $a=0.65 \mu \mathrm{m}$, as described in the text, and is an effective minimum. 


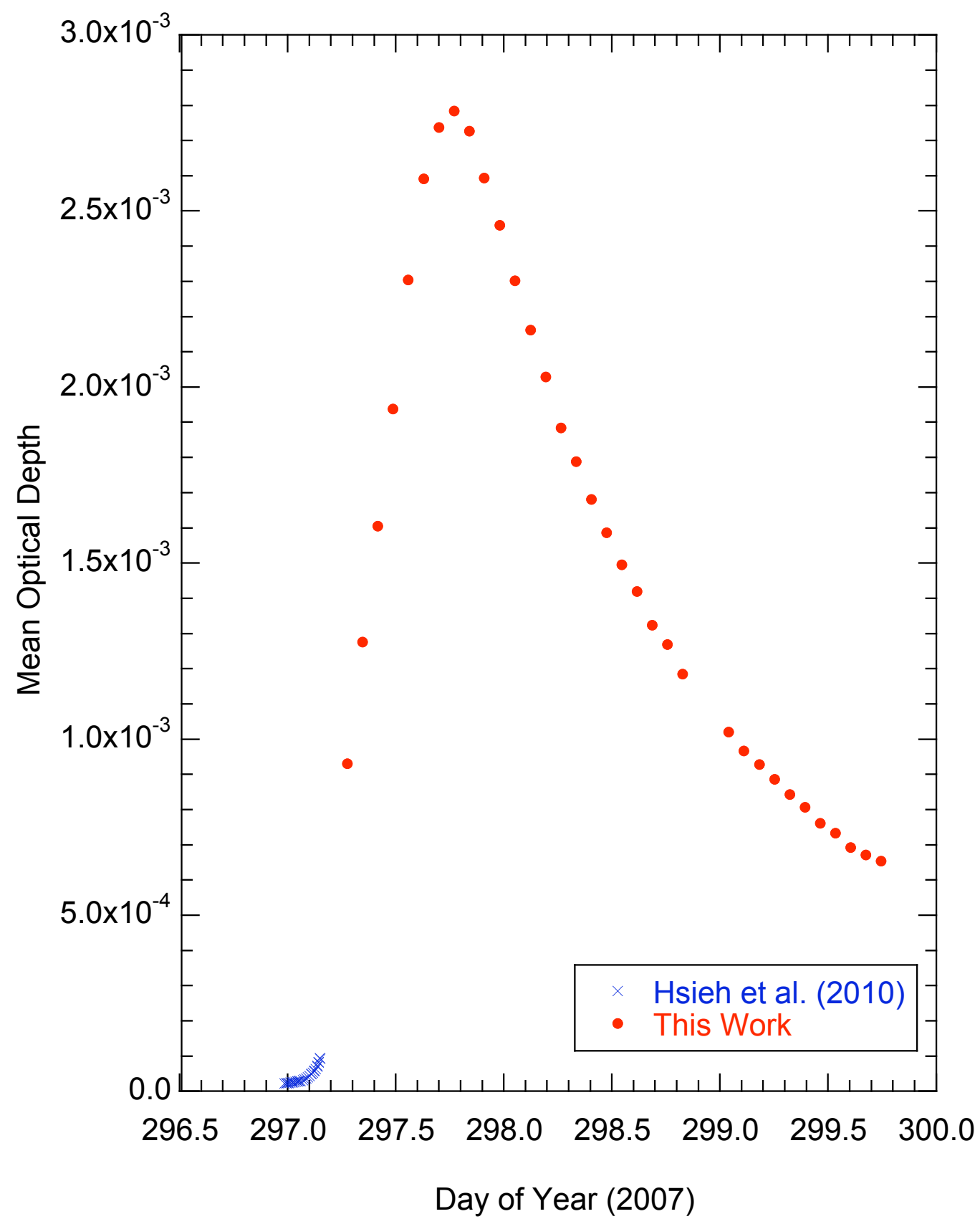

Fig. 7.- Average optical depth versus time near the start of the outburst computed as described in the text. 


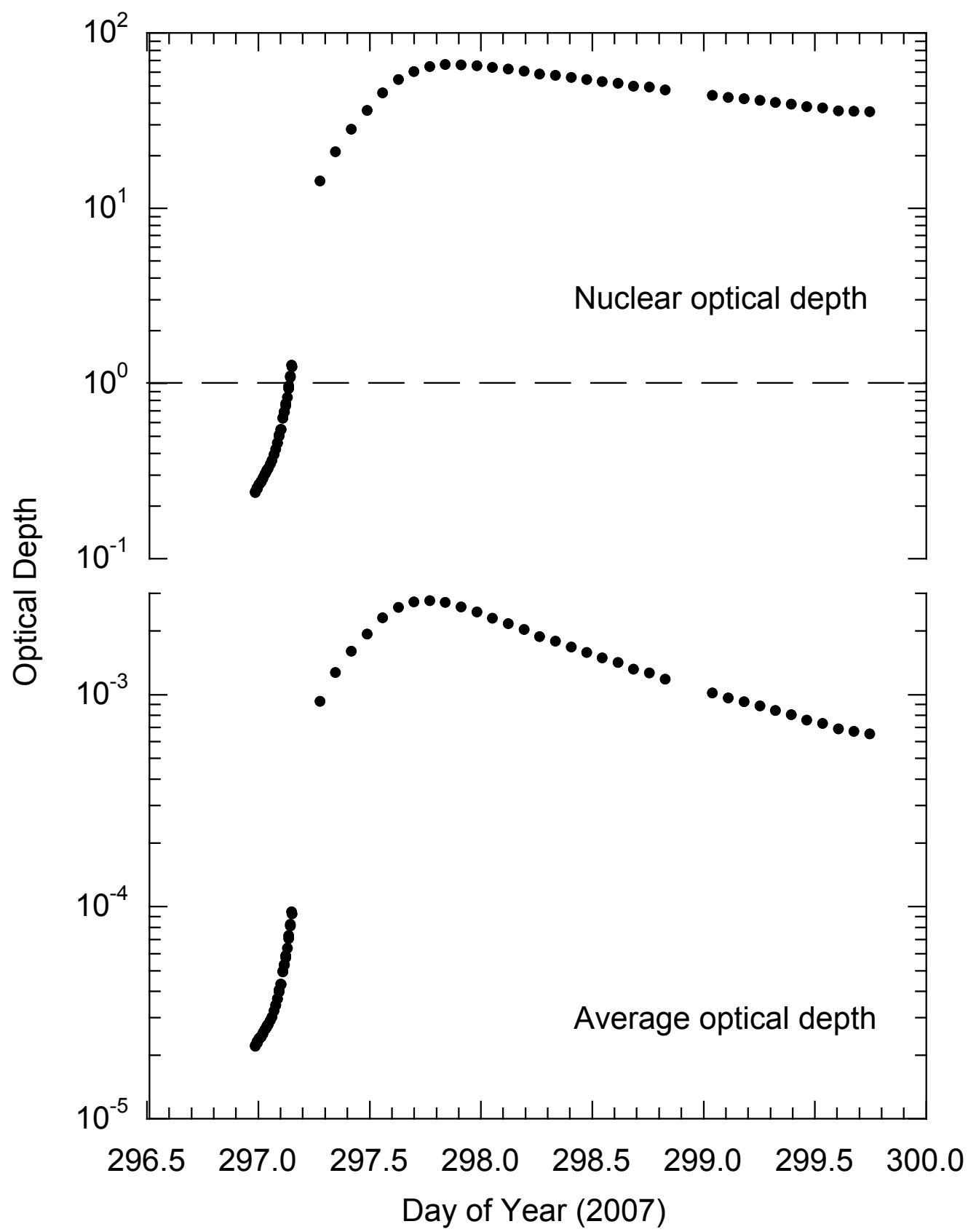

Fig. 8.- Average and peak optical depth versus time. The vertical axis is broken for clarity of presentation, and a horizontal, dashed line shows the region where the coma is optically thick on a line to the center of the nucleus. 